\title{
Nasal versus full face mask for noninvasive ventilation in chronic respiratory failure
}

\author{
G.N. Willson*,\#, A.J. Piper*,\#, M. Norman ${ }^{\Uparrow}$, W.G. Chaseling*,\#, M.A. Milross*,\#, E.R. Collins*,\#, \\ R.R. Grunstein*,\#
}

Nasal versus full face mask for noninvasive ventilation in chronic respiratory failure. G.N. Willson, A.J. Piper, M. Norman, W.G. Chaseling, M.A. Milross, E.R. Collins, R. R. Grunstein. (C) ERS Journals Ltd 2004.

ABSTRACT: This study was undertaken to determine the efficacy of nasal mask (NM) versus full face mask (FFM) for the delivery of noninvasive ventilation (NIV) in subjects with nocturnal hypoventilation.

A total of 16 patients (11 males) were enrolled, all with nocturnal hypoventilation currently treated at home with NIV via pressure preset devices. Subjects underwent full polysomnography on three occasions; on the first night current therapy on NM was reviewed, followed by two experimental studies in randomised order using either NM or FFM. NIV settings and oxygen flow rate were the same under both conditions. Notably, 14 of the 16 subjects required the use of a chinstrap to minimise oral leak.

Apnoea-hypopnoea indices were within normal limits under both conditions $(1.7 \pm 3.4$ $\mathrm{NM}$ versus 1.6 $\pm 2.4 \mathrm{~h}$ FFM). The type of interface did not significantly affect gas exchange during sleep (minimum average arterial oxyhaemoglobin saturation total sleep time 93.4 \pm 2.1 NM versus $92.8 \pm 2.5 \%$ FFM, Delta transcutaneous carbon dioxide nonrapid eye movement sleep to rapid eye movement sleep $(0.58 \pm 0.36 \mathrm{NM}$ versus $0.50 \pm 0.40 \mathrm{kPa}$ FFM). Sleep efficiency was significantly reduced on the FFM $(78 \pm 9$ NM versus $70 \pm 14 \%$ FFM), although arousal indices were comparable under both conditions (15.6 \pm 9.8 NM versus $15.8 \pm 8.8$ h FFM).

Full face masks appear to be as effective as nasal masks in the delivery of noninvasive ventilation to patients with nocturnal hypoventilation. However, a chinstrap was required to reduce oral leak in the majority of subjects using the nasal mask.

Eur Respir J 2004; 23: 605-609.
${ }^{*}$ Centre for Respiratory Failure and Sleep Disorders, Royal Prince Alfred Hospital, ${ }^{\#}$ Woolcock Institute of Medical Research and 'David Read Laboratory, Dept of Medicine, University of Sydney, Sydney, Australia.

Correspondence: G.N. Willson, Centre for Respiratory Failure and Sleep Disorders, Royal Prince Alfred Hospital, Missenden Road, Camperdown NSW 2050 Australia. Fax: 61295157196

E-mail: gnw@galen.med.usyd.edu.au

Keywords: Mask interfaces

mechanical ventilation

noninvasive ventilation

respiratory insufficiency

sleep apnoea syndromes

Received: May 92003

Accepted after revision: November 112003

G.N. Willson and A.J. Piper are consultants to ResMed Ltd. A.J. Piper holds options in ResMed Ltd.
Noninvasive mask ventilatory support is now seen as the treatment of choice in the management of patients with respiratory failure during sleep [1]. Growth in the popularity of this therapy has arisen from improvements in technology, with simple low cost devices designed specifically to provide ventilatory support during sleep now widely available. Along with these advances in machine design has been the development of a variety of both nasal and full face masks (FFM)s for use with such devices.

Traditionally, nasal masks (NM)s have been the most common type of interface used in the home setting to provide noninvasive ventilatory support. However, with use of a NM air may escape out the mouth. Such leaking may not only create uncomfortable side effects for the patient [2], it can also lead to an incomplete response to therapy. Leakage of air out the mouth has been shown to adversely affect sleep quality [3-5], contribute to failure to fully correct nocturnal gas exchange [4] and possibly even increase the work of breathing $[3,6]$, all of which may affect the patient's tolerance and longterm response to therapy.

The purpose of this study was to compare NM and FFM ventilation in patients with nocturnal respiratory failure to determine differences in terms of sleep quality, gas exchange and tolerability. Given that the FFM covers both the nose and mouth, it may reduce leak and more effectively control nocturnal disordered breathing. In addition, this study aimed to determine any differences in pressure settings required between the two types of mask.

\section{Methods}

\section{Patient selection}

Patients with daytime hypercapnic respiratory failure from any cause (restrictive or obstructive disorders) and sleep disordered breathing documented on polysomnography were eligible to be included in this study. Nocturnal hypoventilation was scored in the presence of sustained reductions in arterial oxygen saturation $\left(\mathrm{Sa}_{\mathrm{a}} \mathrm{O}_{2}\right)$ and rise in transcutaneous carbon dioxide $\left(\mathrm{TcCO}_{2}\right)$ accompanied by a reduction in airflow and respiratory effort. Subjects had to be using a noninvasive pressure preset ventilatory support device at home for $\geqslant 3$ months and have demonstrated sustained improvements in gas exchange $(>0.6 \mathrm{kPa}$ reduction in daytime carbon dioxide arterial tension $\left.\left(\mathrm{Pa}_{\mathrm{a}} \mathrm{CO}_{2}\right)\right)$ and symptomatology.

Patients were excluded if they had limited upper limb movement, which would prevent or limit the removal of either the nasal or FFM. Those with significant psychiatric or 
psychological conditions likely to prevent accurate reporting with regard to mask comfort and fit, and those with a history of acute respiratory illness within the last month were also excluded. All patients gave informed consent to participate in the study. The protocol was approved by the Central Sydney Area Health Service ethics committee.

Measurements of anthropometric variables (height/weight), awake arterial blood gases and spirometry were performed in all patients. They then underwent full polysomnography on three occasions. On the first night, current therapy and settings were reviewed. This was followed by two consecutive night studies in randomised order using either a NM or FFM. The maximum time period between the first study (review night) and the subsequent studies was 2 months. Subjects continued to take their normal medications throughout the period of the study. No patient took any hypnotic or antidepressant medication prior to the sleep studies.

\section{Sleep studies}

Full polysomnographic recordings were carried out in the sleep laboratory between 21:00 $\mathrm{h}$ and 07:30 h. Sleep state was monitored using two channels of electroencephalogram (C4/ A1,01/A2 or C3/A2,02/A1), two channels of electro-oculogram (EOG: left outer canthus/right outer canthus), and one channel of submental electromyogram. Monitored breathing variables included chest wall and abdominal motion, diaphragm electromyogram, $\mathrm{Sa}, \mathrm{O}_{2}$ (Biox3700e; Ohmeda, Boulder, CO, USA), $\mathrm{TcCO}_{2}$ (Tina; Radiometer, Copenhagen, Denmark) and nasal airflow using a pressure transducer (AutoSet ${ }^{\mathrm{TM}}$; ResMed Inc, Sydney, Australia). Electrocardiograms were measured continuously in all patients. All variables were recorded on a 16 channel polygraph (Sleepwatch ${ }^{\mathrm{TM}}$; Compumedics, Melbourne, Australia).

Sleep state was scored in $30 \mathrm{~s}$ epochs according to standard criteria [7]. To facilitate analysis, sleep stages 1 and 2 were combined, as were stages 3 and 4 (slow wave sleep (SWS)). Sleep stages were expressed as a percentage of total sleep time (TST). Sleep efficiency was defined as the percentage of the total recording time that was scored as sleep. Arousal was defined as an awakening for $>3 \mathrm{~s}$ [8].

Respiratory events on bilevel ventilatory support were scored in the presence of reduced thoraco-abdominal wall motion for $\geqslant 10 \mathrm{~s}$ with $\geqslant 3 \%$ decrease in oxygen saturation or an electroencephalogram arousal. The number of events per $h$ of sleep were calculated and expressed as an apnoeahypopnoea index (AHI). The minimum average $\mathrm{Sa}_{2} \mathrm{O}_{2}$ (minimum average $\mathrm{Sa}_{\mathrm{a}} \mathrm{O}_{2}$ TST) was calculated as the mean of the minimum value for $\mathrm{Sa}_{2} \mathrm{O}_{2}$ in each $30 \mathrm{~s}$ epoch. The minimum $\mathrm{Sa}_{2} \mathrm{O}_{2}$ was the lowest value recorded during sleep. The change in $\mathrm{TcCO}_{2}$ whilst receiving nocturnal ventilatory support was analysed. The maximum delta change in $\mathrm{TcCO}_{2}$ from nonrapid eye movement sleep (NREM) to rapid eye movement sleep (REM) was calculated for each patient and the worst case was reported. Allowance was made for any drift in $\mathrm{TcCO}_{2}$ using the authors' previously documented method [9].

Subjects used the bilevel ventilatory support device, which had previously been prescribed and used at home. The mode, pressure settings and inspiratory duration were set to those optimised on the review study and remained unchanged at the subsequent mask trials (table 1). The devices were set in the spontaneous/timed mode in the presence of central apnoea or profound hypoventilation with a failure to cycle to inspiration in the spontaneous mode. Expiratory positive airway pressure (EPAP) was titrated to abolish upper airway obstruction. This was evidenced by repetitive failure of the device to cycle to inspiration accompanied by maintenance of respiratory effort. Inspiratory positive airway pressure was used to augment tidal volume and reverse nocturnal hypoventilation evidenced by a reduction in respiratory effort and a rise in $\mathrm{TcCO}_{2}$. A chinstrap was added if there was worsening machine-patient synchronisation with a loss of thoracoabdominal motion and/or deterioration in gas exchange in the presence of witnessed leak. Supplemental oxygen was added during the review night if $\mathrm{Sa}, \mathrm{O}_{2}$ persisted $<90 \%$ during sleep, once settings were optimised. This was entrained into the circuit via a connector located adjacent to the flow generator. The flow rate of oxygen used was unchanged during both the nasal and FFM nights.

\section{Interface acclimatisation}

All subjects enrolled in the study were using NM ventilation at home. Table 1 lists the masks used by individual

Table 1. - Settings used for nocturnal ventilation

\begin{tabular}{|c|c|c|c|c|c|c|c|c|}
\hline Patient & Device & Mode & EPAP $\mathrm{cmH}_{2} \mathrm{O}$ & IPAP $\mathrm{cmH}_{2} \mathrm{O}$ & Rate bpm & Maximum IPAP s & Oxygen flow rate $\mathrm{L} \cdot \mathrm{min}^{-1}$ & Nasal mask \\
\hline 1 & VPAP II & $\mathrm{S}$ & 6 & 15 & NA & 1.5 & 2 & Bubble \\
\hline 2 & VPAP II & $\mathrm{S}$ & 14 & 20 & NA & 1.4 & 2 & Goldseal \\
\hline 3 & REM+Duo & ST & 5 & 15 & 15 & 1.4 & Nil & Bubble \\
\hline 4 & VPAP II & $\mathrm{S}$ & 7 & 17 & NA & 1.45 & 1 & Bubble \\
\hline 5 & VPAP II & $\mathrm{S}$ & 7 & 16 & NA & 1.5 & Nil & Bubble \\
\hline 6 & VPAP II & $\mathrm{S}$ & 6 & 16 & NA & 1.2 & 2 & Bubble \\
\hline 7 & BIPAP & $\mathrm{S}$ & 5 & 14 & NA & NA & Nil & Standard \\
\hline 8 & VPAP II & $\mathrm{S}$ & 13 & 17 & NA & 3.0 & 2 & Mirage \\
\hline 9 & BIPAP & ST & 4 & 16 & 18 & NA & 1 & Bubble \\
\hline 10 & VPAP II & ST & 5 & 20 & 20 & 1.25 & Nil & Bubble \\
\hline 11 & VPAP II & $\mathrm{S}$ & 13 & 19 & NA & 2.1 & 1.5 & Bubble \\
\hline 12 & Horizon & $S$ & 10 & 16 & NA & NA & 2 & Standard \\
\hline 13 & REM+Duo & ST & 6 & 15 & 22 & 1.23 & Nil & Bubble \\
\hline 14 & BIPAP & $\mathrm{S}$ & 10 & 18 & NA & NA & 1 & Standard \\
\hline 15 & VPAP II & ST & 4 & 14 & 19 & 1.3 & Nil & Bubble \\
\hline 16 & VPAP II & $\mathrm{S}$ & 6 & 13 & NA & 1.35 & 1 & Mirage \\
\hline Mean & & & 7.6 & 16.3 & 18.8 & 1.6 & 1.6 & \\
\hline
\end{tabular}

Devices and nasal masks include: VPAP II (ResMed, Sydney, Australia), REM+Duo (Nelcor Puritan-Bennett, Villers-Les-Nancy, France), BIPAP (Respironics, Murrysville, PA, USA), Horizon (DeVilbuss, Somerset, NJ, USA), Bubble, Standard, Mirage (ResMed, Sydney, Australia), Goldseal (Respironics, Murrysville, PA USA); S: Spontaneous mode; ST: spontaneous timed mode; EPAP: expiratory positive airway pressure; IPAP: inspiratory positive airways pressure; bpm: breaths per min; NA: not applicable. 
subjects. On the routine review night and the NM night subjects used the mask that had been fitted and tested during previous polysomnography. All participants used a commercially available FFM (Mirage Series 1; ResMed, Sydney, Australia). Subjects received information concerning the FFM, such as its features, method of attachment and method of removal, including quick release strap. Daytime practice sessions were undertaken to ensure proficiency with attachment and removal. Prolonged usage of the mask during the day $(\sim 0.5 \mathrm{~h})$ with the patient relaxed and comfortable was a prerequisite prior for continuing the protocol. On the morning following each study subjects used a visual analogue scale to rate the level of perceived leak and comfort. Subjects were asked to rate "How much did the mask leak?" from 0 (no leak at all) to 10 (leaked constantly) or anywhere in between and "Was the mask comfortable?" from 0 (very comfortable) to 10 (very uncomfortable) or anywhere in between, in relation to the interface used on the previous night.

\section{Statistical analysis}

All values are shown as mean \pm SD unless stated otherwise. The effect of treatment on AHI, arousals, sleep architecture and gas exchange was tested using paired t-tests. A p-value of $<0.05$ was considered significant.

\section{Results}

\section{Patients}

A total of 16 subjects (11 males) with documented nocturnal hypoventilation (minimum $\mathrm{Sa}, \mathrm{O}_{2}, 57 \pm 27 \%$ on diagnostic polysomnography) during sleep were studied. The patient's clinical characteristics including age, anthropometric data, body mass index (BMI), spirometry and arterial blood gases are shown in table 2. The mean age of the patients was $61 \pm 11 \mathrm{yrs}$ (range $39-80 \mathrm{yrs}$ ). Mean BMI was $32 \pm 12 \mathrm{~kg} \cdot \mathrm{m}^{-2}$, and varied widely depending on the aetiology of respiratory failure (range $18.5-53.2 \mathrm{~kg} \cdot \mathrm{m}^{-2}$ ). All subjects were in hypercapnic respiratory failure with a mean $\mathrm{Pa}, \mathrm{CO}_{2}$ of $10.1 \pm 3.1 \mathrm{kPa}$ prior to the initiation of noninvasive ventilation (NIV). NIV had resulted in substantial improvements in gas exchange with a reduction in mean daytime $P a, \mathrm{CO}_{2}$ to $7.0 \pm 0.8 \mathrm{kPa}$. Subjects had been using NIV at home for a median of 16 months (range 3-70 months) and all had experienced an improvement in their presenting symptomatology.

The underlying cause of respiratory failure was obesity hypoventilation syndrome (six patients), chest wall deformity (five patients), neuromuscular disease (three patients), chronic airflow limitation (two patients), obstructive sleep apnoea (one patient) and central alveolar hypoventilation (one patient) (table 2). All subjects were in a stable clinical state at the time of enrolment into the study.

The ventilatory support device, settings, oxygen flow rate and NM used during NIV are shown in table 1. Five subjects required the use of the Spontaneous Timed mode due to the presence of central apnoea or profound hypoventilation. Fourteen of the sixteen subjects routinely used a chinstrap (Bluegum Sewing and Craft, Canberra, Australia) at home to minimise oral leak whilst using the NM. Altogether, nine of the 16 subjects used supplemental oxygen in addition to bilevel ventilatory support, with flow rates between 1 and $2 \mathrm{~L} \cdot \mathrm{min}^{-1}$.

\section{Sleep Studies}

The sleep study data are summarised in table 3. The AHI were within normal limits using both the NM and FFM (1.7 $\pm 3.4 \mathrm{NM}$ versus $1.6 \pm 2.4 \mathrm{~h}$ FFM (nonsignificant)). In patient 5 , an AHI of 12 events $\cdot h^{-1}$ occurred while using the NM, while patient 15 had an AHI of 8 events $\cdot h^{-1}$ on the FFM. Both subjects were diagnosed with neuromuscular disease. The persistent respiratory events during NIV appeared to be predominantly due to residual upper airway obstruction or persistent REM hypoventilation associated with leak.

The type of interface used did not significantly affect oxygenation during TST (minimum average $S \mathrm{a}, \mathrm{O}_{2}$ TST 93.4 \pm 2.1 NM versus $92.8 \pm 2.5 \%$ FFM $(\mathrm{p}=0.09)$ ). However, when considered separately, minimum average $\mathrm{Sa}, \mathrm{O}_{2}$ during

Table 2. - Patient characteristics

\begin{tabular}{|c|c|c|c|c|c|}
\hline \multirow[t]{2}{*}{ Patient } & \multirow[t]{2}{*}{ Age yrs } & \multirow[t]{2}{*}{ Diagnostic group } & \multirow[t]{2}{*}{$\mathrm{BMI} \mathrm{kg} \cdot \mathrm{m}^{-2}$} & \multicolumn{2}{|c|}{ Spirometry } \\
\hline & & & & FEV1 L BTPS & FVC L BTPS \\
\hline 1 & 54 & CAL & 20 & 0.6 & 2.3 \\
\hline 2 & 68 & OHS & 42.7 & 1.35 & 2.09 \\
\hline 3 & 73 & $\mathrm{Nm}$ & 24 & 1.85 & 2.61 \\
\hline 4 & 65 & OHS & 39.6 & 1.79 & 2.38 \\
\hline 5 & 62 & $\mathrm{Nm}$ & 29 & 1.4 & 1.56 \\
\hline 6 & 64 & OHS/CAL & 35.7 & 0.57 & 0.99 \\
\hline 7 & 53 & $\mathrm{CAH}$ & 32.1 & 3.3 & 4.45 \\
\hline 8 & 70 & OHS & 52.2 & 1.67 & 2.02 \\
\hline 9 & 57 & $\mathrm{CW}$ & 22.6 & 0.47 & 0.52 \\
\hline 10 & 39 & CW & 20.3 & 0.71 & 0.73 \\
\hline 11 & 43 & CW/OSA & 28.9 & 1.4 & 2.23 \\
\hline 12 & 51 & OHS & 53.2 & 1.45 & 2.05 \\
\hline 13 & 55 & CW & 18.5 & 0.85 & 1.3 \\
\hline 14 & 69 & OHS & 48.7 & 1.45 & 2.04 \\
\hline 15 & 80 & $\mathrm{Nm}$ & 18.9 & 0.5 & 0.81 \\
\hline 16 & 73 & $\mathrm{CW}$ & 25 & 0.4 & 0.49 \\
\hline Mean \pm SD & $61 \pm 11$ & & $32 \pm 12$ & $1.2 \pm 0.7$ & $1.8 \pm 1.0$ \\
\hline
\end{tabular}

BMI: body mass index; FEV1: forced expiratory volume in one second; FVC: forced vital capacity; L BTPS: litres body temperature and pressure saturated; CAL: chronic airflow limitation; OHS: obesity-hypoventilation syndrome; Nm: neuromuscular disease; OSA: obstructive sleep apnoea; $\mathrm{CAH}$ : central alveolar hypoventilation; $\mathrm{CW}$ : chest wall deformity. 
Table 3. - Sleep study data

Nasal mask Full face mask p-value

\begin{tabular}{lccc}
\hline Patients n & 16 & 16 & \\
TST min & $339 \pm 56$ & $300 \pm 79$ & $<0.05$ \\
Sleep efficiency \% & $78 \pm 9$ & $70 \pm 14$ & $<0.05$ \\
Sleep latency min & $20 \pm 24$ & $21 \pm 13$ & NS \\
Stage 1/2 \% TST & $70 \pm 12$ & $67 \pm 12$ & NS \\
Slow wave sleep \% TST & $12 \pm 11$ & $11 \pm 10$ & NS \\
REM \%TST & $18 \pm 8$ & $22 \pm 8$ & $<0.02$ \\
AHI events· ${ }^{-1}$ & $1.7 \pm 3.4$ & $1.6 \pm 2.4$ & NS \\
Minimum average $\mathrm{Sa}_{\mathrm{a}, \mathrm{O}_{2}}$ & & & \\
$\quad$ TST \% & $93.4 \pm 2.1$ & $92.8 \pm 2.5$ & 0.09 \\
$\quad$ NREM \% & $93.6 \pm 2.0$ & $93.0 \pm 2.4$ & $<0.05$ \\
$\quad$ REM \% & $92.4 \pm 2.9$ & $92.0 \pm 3.5$ & $\mathrm{NS}$ \\
Arousal index arousals $\cdot \mathrm{h}^{-1}$ & $15.6 \pm 9.8$ & $15.8 \pm 8.8$ & $\mathrm{NS}$ \\
\hline
\end{tabular}

Results expressed as mean \pm SD unless otherwise stated. TST: total sleep time; REM: rapid eye movement sleep; AHI: apnoea-hypopnoea index; $\mathrm{Sa}_{2} \mathrm{O}_{2}$ : arterial oxyhaemoglobin saturation; NREM: nonrapid eye movement sleep; NS: nonsignificant.

NREM was statistically lower during the FFM night (minimum average $\mathrm{Sa}, \mathrm{O}_{2} \mathrm{NREM} 93.6 \pm 2.0 \mathrm{NM}$ versus $93.0 \pm 2.4 \%$ FFM $(\mathrm{p}<0.05))$, although this mean difference would not have been of clinical significance. Minimum average $\mathrm{Sa}_{\mathrm{a}} \mathrm{O}_{2}$ during REM was unchanged under both conditions (minimum average $\mathrm{Sa}, \mathrm{O}_{2}$ REM 92.4 \pm 2.9 NM versus $92.0 \pm 3.5 \%$ FFM (nonsignificant)). The subgroup analysis showed that as a group the reduction in overnight $S \mathrm{a}, \mathrm{O}_{2}$ was confined to those receiving supplemental oxygen (minimum average $S a, O_{2}$ TST $93.6 \pm 2.1 \mathrm{NM}$ versus $92.2 \pm 2.9 \%$ FFM $(\mathrm{p}<0.01)$ ) versus those not receiving supplemental oxygen with NIV (minimum average $\mathrm{Sa}_{\mathrm{a}} \mathrm{O}_{2}$ TST $93.1 \pm 2.2 \mathrm{NM}$ versus $93.5 \pm 2.0 \%$ FFM (nonsignificant)). Distinct rises in $\mathrm{TcCO}_{2}$ were often seen during REM sleep under both conditions. The change in Delta $\mathrm{TcCO}_{2}$ NREM-REM was similar under both conditions (Delta $\mathrm{TcCO}_{2} \quad$ NREM-REM $0.58 \pm 0.36 \quad \mathrm{NM}$ versus $0.50 \pm 0.40 \mathrm{kPa}$ FFM (nonsignificant)). Patient 3 had a $0.40 \mathrm{kPa}$ fall in REM $\mathrm{TcCO}_{2}$ on the FFM as opposed to a $0.93 \mathrm{kPa}$ increase on the NM. Patient 15 had a rise of $1.06 \mathrm{kPa}$ in $\mathrm{TcCO}_{2}$ during REM on the FFM versus no change on the NM.

The latency to sleep was the same under both conditions ( $20 \pm 24 \mathrm{NM}$ versus $21 \pm 13 \mathrm{~min}$ FFM (nonsignificant)). TST was reduced from $339 \pm 56 \mathrm{~min}$ on the NM to $300 \pm 79 \mathrm{~min}$ on the FFM $(\mathrm{p}<0.05)$. Sleep efficiency was also reduced on the FFM $(78 \pm 9$ NM versus $70 \pm 14 \%$ FFM $(\mathrm{p}<0.05))$. This decrement in sleep efficiency was primarily due to four subjects with reductions in sleep efficiency of $>15 \%$ (patients 2, 6, 8 and 13). Despite these changes, arousal indices were

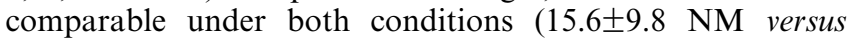
$15.8 \pm 8.8 \mathrm{~h}$ FFM (nonsignificant)). There was no change in the proportion of stage $1 / 2$ and SWS, although there was an increase in proportion of REM sleep whilst using the FFM (REM \%TST $18 \pm 8$ NM versus $22 \pm 8 \%$ FFM ( $\mathrm{p}=0.02)$ ). There was no change in the absolute duration of REM sleep (REM $63 \pm 35 \mathrm{NM}$ versus $67 \pm 35$ min FFM (nonsignificant)).

\section{Subjective responses}

There was a trend toward a subjective increase in comfort on the NM with a visual analogue score of $1.8 \pm 1.6$ versus $3.1 \pm 2.6$ on the FFM ( $\mathrm{p}=0.07)$. This was accompanied by the perception of increased leak on the FFM 5.2 \pm 3.2 versus $2.0 \pm 2.0$ on the NM $(\mathrm{p}<0.01)$.

\section{Discussion}

The purpose of this study was to compare the impact of NM with FFM use on sleep quality and gas exchange in subjects using NIV. In addition, the authors wished to ascertain subjective responses regarding comfort and leak. The data shows that the FFM used was equally effective as a NM in maintaining nocturnal gas exchange and preventing sleep disordered breathing in patients with known nocturnal hypoventilation syndromes, without the need to alter pressure settings. During the night the FFM was used, sleep efficiency was reduced, although the percentage of REM sleep time increased. No differences in arousal indices between the two nights were observed. Notably, 14 of the 16 subjects required the use of a chinstrap to minimise oral leak. Subjectively, patients felt the NM was slightly more comfortable to use, with fewer leaks compared with using the FFM.

Traditionally, NMs have been the primary means of providing long-term nocturnal ventilatory support for patients with chronic respiratory failure. However, mouth leaks during sleep are common, [3, 5], and known to be associated with arousal, reduced sleep efficiency and poorer sleep quality [3-5]. In addition, loss of volume out the mouth results in reduced ventilation and less effective control of nocturnal carbon dioxide levels [4]. By covering the mouth, FFMs should reduce leak, thereby improving gas exchange and sleep quality. However, apart from a reduction in TST and sleep efficiency, no difference in sleep architecture or arousals from sleep between the two interfaces was found, nor were clinically significant differences in gas exchange identified between the two nights. This may appear surprising, given that facemasks should prevent mouth leak and its consequences. However, an important methodological consideration is that 14 of the 16 subjects in this study were using a chinstrap with their NM to minimise mouth leak. It is possible that chinstrap use during the NM night was in fact a very effective strategy in minimising leak and therefore biased the current study against seeing any significant benefit of the FFM over the NM. Although chinstraps have been advocated as a means of reducing mouth leak [10] there is only scant data in the literature confirming their effectiveness [11]. Interestingly, in the current study, TST, percentage of REM sleep and the arousal index during the NM night with chinstrap use was surprisingly similar to that reported by TESCHLER et al. [4] during a night when subjects using nocturnal ventilatory support were asked to tape their mouths closed to minimise mouth leak. Although the results from the current study and TESCHLER et al. [4] are not directly comparable, it does raise the possibility that the chinstraps used with the intent of reducing leak were effective in minimising mouth leaks and its consequences. If this were the case then any benefits of FFM over NM would have been minimised. Further studies are needed to investigate this possibility.

During the night of FFM use, patients reported sleeping less well and demonstrated lower sleep efficiency. A major factor that could have influenced this result is that patients were already adapted to the NM and therefore likely to find this interface more comfortable when compared with another type of interface during a single night of use. The FFM was fitted and trialled on the afternoon of the study, but patients were not familiar with using it during sleep. A more prolonged acclimatisation period including nocturnal use might have enabled patients to fit and adjust the mask more effectively. This may have reduced leak from around the mask and improved comfort. With more time awake, patients would have had a greater opportunity to be aware of leaks from the FFM and therefore be able to report them. The authors' clinical observations and those of others is that 
mouth leaks with the NM occur primarily during sleep periods $[3,12]$ and therefore are frequently not perceived by the patient. The finding of a trend towards increased comfort on the NM is supported by a number of other studies, which have shown improved breathing comfort on the NM [13] and a preference for NM [14] over FFM usage.

Alterations in machine settings were not needed when interfaces were changed. However, a small reduction in oxygen saturation during sleep was found in those patients receiving supplemental oxygen with their bilevel ventilatory support with the FFM. This was most likely due to the characteristics of the FFM used. The expiratory ports in the FFM used have an expiratory gas flow of $27 \mathrm{~L} \cdot \mathrm{min}^{-1}$ at an EPAP of $5 \mathrm{cmH}_{2} \mathrm{O}$. In contrast, the NMs used had lower expiratory flow rates of $19-21 \mathrm{~L} \cdot \mathrm{min}^{-1}$ at the same expiratory pressure. It is possible that the increased flow from the FFM expiratory port may have washed out supplemental oxygen more efficiently than the NM, giving a small reduction in the inhaled inspiratory oxygen fraction, resulting in the small reduction in $\mathrm{Sa}, \mathrm{O}_{2}$ that was observed. This finding is a potentially important consideration when changing interfaces in patients requiring supplemental oxygen during NIV.

\section{Conclusions}

Full face masks appear to be as effective as nasal masks in the delivery of noninvasive ventilation to patients with nocturnal hypoventilation syndromes. Although it was not necessary to adjust pressures to accommodate the change in interface, the potential for reduced oxygenation at a given oxygen flow rate and possibly reduced comfort with the full face mask should be considered with the change. Full face masks may be advantageous in patients who are unable to tolerate a nasal mask due to nasal pathology, or where clinically significant mouth leaks persist despite the use of a chinstrap. With the ongoing development of full face masks, both clinicians and patients will have greater choices when it comes to choosing interfaces for nocturnal ventilatory support. Further studies are needed to access the long-term efficacy of these interfaces and determine if the choice of interface plays an important role in patient outcomes.

Acknowledgements. The authors greatly appreciate the technical support of the staff at the Centre for Respiratory Failure and Sleep Disorders, Royal Prince Alfred Hospital, in carrying out the sleep studies.

\section{References}

1. Mehta S, Hill NS. Noninvasive ventilation. Am J Respir Crit Care Med 2001; 163: 540-577.

2. Richards GN, Cistulli PA, Unger RG, Berthon-Jones M, Sullivan CE. Mouth leak with nasal continuous positive airway pressure increases nasal airway resistance. $\mathrm{Am}$ J Respir Crit Care Med 1996; 154: 182-186.

3. Meyer TJ, Pressman MR, Benditt J, et al. Air leaking through the mouth during nocturnal nasal ventilation: effect on sleep quality. Sleep 1997; 20: 561-569.

4. Teschler H, Stampa J, Ragette R, Konietzko N, BerthonJones M. Effect of mouth leak on effectiveness of nasal bilevel ventilatory assistance and sleep architecture. Eur Respir J 1999; 14: 1251-1257.

5. Bach J, Robert D, Leger P, Langevin B. Sleep fragmentation in kyphoscoliotic individuals with alveolar hypoventilation treated by NIPPV. Chest 1995; 107: 1552-1558.

6. Carrey Z, Gottfried SB, Levy RD. Ventilatory muscle support in respiratory failure with nasal positive pressure ventilation. Chest 1990; 97: 150-158.

7. Rechtschaffen A, Kales A. A manual of standardized terminology, techniques and scoring system for sleep stages of human subjects. National Institutes of Health, Washington DC, 1968.

8. Bonnet $\mathrm{MH}$, Carley D, Carskadon $\mathrm{M}$, et al. EEG arousals: scoring rules and examples. A preliminary report from the Sleep Disorders Atlas Task Force of the American Sleep Disorders Association. Sleep 1992; 15: 174-184.

9. Milross MA, Piper AJ, Norman M, et al. Low-flow oxygen and bilevel ventilatory support: Effects on ventilation during sleep in cystic fibrosis. Am J Respir Crit Care Med 2001; 163: 129-134.

10. Piper AJ, Willson GN. Nocturnal nasal ventilatory support in the management of daytime hypercapnic respiratory failure. Aust J Physiother 1996; 42: 17-29.

11. Gonzalez J, Sharshar T, Hart N, Chadda K, Raphael JC, Lofaso F. Airleaks during mechanical ventilation as a cause of persistent hypercapnia in neuromuscular disorders. Intensive Care Med 2003; 29: 596-602.

12. Gonzalez MM, Parreira VF, Rodenstein DO. Non-invasive ventilation and sleep. Sleep Med Rev 2002; 6: 29-44.

13. Mortimore IL, Whittle AT, Douglas NJ. Comparison of nose and face mask CPAP therapy for sleep apnoea. Thorax 1998; 53: 290-292.

14. Navalesi P, Fanfulla F, Frigerio P, Gregoretti C, Nava S. Physiologic evaluation of noninvasive mechanical ventilation delivered with three types of masks in patients with chronic hypercapnic respiratory failure. Crit Care Med 2000; 28: $1785-1790$. 